\title{
Relational Knowledge Discovery in Databases
}

\author{
Hendrik Blockeel and Luc De Raedt \\ Katholieke Universiteit Leuven \\ Department of Computer Science \\ Celestijnenlaan 200A \\ 3001 Heverlee \\ e-mail: \{Hendrik.Blockeel, Luc.DeRaedt\}@cs.kuleuven.ac.be
}

\begin{abstract}
In this paper, we indicate some possible applications of ILP or similar techniques in the knowledge discovery field, and then discuss several methods for adapting and linking ILP-systems to relational database systems. The proposed methods range from "pure ILP" to "based on techniques originating in ILP". We show that it is both easy and advantageous to adapt ILP-systems in this way.
\end{abstract}

\section{Introduction}

It is common knowledge that in the machine learning field, ILP has turned out to be very useful for classification problems in structured domains. Similar problems can be found in the knowledge discovery field. For instance, finding integrity constraints holding in a database closely corresponds to finding classification rules with ILP, as we will show later on in this paper.

In the past, research on knowledge discovery in databases (KDD) has focused mainly on propositional techniques; this implies that relationships between the attributes of one tuple can be found, but no relationships between several tuples of one or more relations. It seems that this is an important limitation, since problems such as finding integrity constraints cannot be solved using propositional techniques (at least not in an easy way).

In this paper, we show that some problems in knowledge discovery are closely related to typical ILP classification problems, and that therefore ILP or ILP-like methods are useful to solve them (section 2). We then show in section 3 how ILP can be adapted for this kind of problems, and eventually (in section 4) arrive at an algorithm for KDD that searches for regularities expressed in relational algebra instead of logic. Such an algorithm strictly does not fall in the class of ILP algorithms but it does use techniques based on ILP. In section 5 , finally, we present some conclusions.

\section{What ILP can offer to relational databases}

In the machine learning field, the advantages and disadvantages of ILP vs. other techniques are well-known, and the subject of hot debates. Typical for nonILP systems is that they try to find rules involving several attributes of an 
example. Each example is described using a fixed number of attributes for which values are given. The representational power of these techniques corresponds to that of propositional logic. Therefore, we will also use the term "propositional techniques".

When rules have to be found that involve several examples, or when the examples do not have a fixed number of attributes, these propositional techniques are no longer applicable, as they lack the required representational power. ILP systems, on the other hand, can represent this kind of data and rules, since they use first order predicate logic as a representation formalism, which is more powerful.

If we translate these properties to database terminology, then we can say that propositional systems find rules involving one relation, while ILP systems can also find rules involving several relations.

As a database typically contains more than one relation, it seems clear that a lot of interesting rules are simply not considered when using propositional techniques. Nevertheless, research in the data mining community has focused mainly on these techniques (see e.g. [5]). Finding inter-relation rules can sometimes be simulated by computing the universal relation (i.e. joining all the relations into one relation), but this may be a very costly operation, and the universal relation can be very large. For this reason, it seems that ILP offers interesting opportunities to data mining.

We will now analyze in more detail one problem where ILP is useful for KDD, and on which our group is currently working. We address the following questions: in what way is some relation $R$ related to a set of relations $\mathcal{R}=\left\{R_{1}, \ldots, R_{n}\right\}$ ? Can $R$ be computed from $\mathcal{R}$, and if so, what is the exact way of computing it? If it cannot, are there any other interesting relationships between $R$ and $\mathcal{R}$ ?

These questions are interesting because if $R$ can be expressed in terms of $\left\{R_{1}, \ldots, R_{n}\right\}$, then it is redundant; instead of listing it explicitly, it is probably better to define it in terms of the other relations. If $R$ cannot be expressed exactly in terms of $R_{1}, \ldots, R_{n}$, it may still be interesting to find some expression that defines a (preferably large) subset of $R$ in terms of the other relations; in that case, we could say the expression is an overly specific description of the relation $R$. Analogously, an overly general description of $R$ (an expression that computes a superset of $R$ ) may be of interest, if a correct one cannot be found. Such relationships may not enable the system to compute $R$ from $R_{1}, \ldots, R_{n}$, but they do impose certain integrity constraints on the database. They identify new relations $R^{\prime}$, computed from $R_{1}, \ldots, R_{n}$, for which an inclusion constraint holds between $R$ and $R^{\prime}$ (in one direction or another).

We will from now on use the following terminology to distinguish relations that are computed from other relations, from those that are defined by listing all their tuples.

Definition 1. A relation is defined extensionally if it is defined by exhaustively listing all its tuples.

Definition 2. A relation is defined intensionally if it is defined in terms of other relations by a relational algebra expression. 
In relational databases, relations defined extensionally are usually called $t a-$ bles, while relations defined intensionally are called views. Intensional definitions have the advantage that some redundancy is removed, and possible inconsistencies are avoided; on the other hand, answering queries about the relation becomes more expensive because (part of) the relation has to be computed instead of being retrieved from storage. In many cases, an intensional definition will nevertheless be preferred. However, finding such an intensional definition requires establishing the correct relationship between the relation and other relations, and expressing it in some way (e.g. relational algebra or SQL).

A theoretical answer to whether $R$ can at all be expressed in terms of a set of relations $\mathcal{R}$ using the relational algebra, was given years ago, see for instance [10]. However, there does not seem to be an easy way of computing the answer to this question (i.e. one that is significantly faster than just trying to find such an expression and eventually failing if none exists). Therefore, the algorithms we will consider later on in this text will not try to check beforehand whether an intensional definition of a relation exists or not, but just hope there is one, and try to find it.

\section{Corresponding problems in ILP}

Two settings can be distinguished in ILP. In the classical setting, logical clauses are induced that together form a complete and consistent hypothesis defining a concept. Formally, if $H$ is the induced hypothesis, $B$ is background knowledge that may be available, and $E^{+}$and $E^{-}$are sets of positive and negative examples, then the following two statements must hold:

$$
\begin{aligned}
& \forall e^{+} \in E^{+}: H \cup B \models e^{+} \\
& \nexists e^{-} \in E^{-}: H \cup B \models e^{-}
\end{aligned}
$$

The hypothesis can then be used for classification of unseen examples. Most ILP systems (e.g. Progol [9], FOIL [11]) use this setting.

Another setting is what we call characteristic ILP. Clauses are induced that are valid for the given data; this means that there should not exist a substitution for which the clause is false. Formally, if $c$ is a clause, $B$ is the background knowledge and $E$ a set of examples (we do not distinguish positive and negative examples in this setting), then $c$ is valid iff

$$
c \text { is true in } \mathcal{M}(B \cup E)
$$

(where $\mathcal{M}(\mathcal{B} \cup \mathcal{E})$ denotes the minimal Herbrand model of $B \cup E$ ).

Every clause describes the data in some way, and the set of all valid clauses forms a maximally informative description of the data. ILP systems using this setting exist (e.g. ClaUdien[1], ICL[2]) but are far less numerous than systems using the classical setting.

There is a straightforward relationship between finding an intensional definition for a relation, and classification of tuples. If $R$ is considered to represent some class of tuples $\left(v_{1}, \ldots, v_{k}\right)$, then the expression of $R$ in terms of $\left\{R_{1}, \ldots, R_{n}\right\}$ 
gives a means of deciding whether a specific tuple $\left(v_{1}, \ldots, v_{k}\right)$ belongs to $R$ (i.e. classifying the tuple), using only the information in the relations $R_{1}, \ldots, R_{n}$. We can therefore conclude that the problem of finding intensional definitions is closely related to the classification setting within ILP. ${ }^{1}$

On the other hand, if an intensional definition cannot be found, but the expressions that are induced are considered as integrity constraints, then we would like to find as many such constraints as possible (in order to describe the allowed database instance as precisely as possible). This corresponds to the characteristic ILP setting.

We have hereby established a link between typical ILP problems and finding intensional definitions or integrity constraints in databases. From this we can expect that ILP can easily be adapted for the tasks considered. It is also clear that the kind of relationships discussed here cannot be discovered using propositional techniques, as they involve several relations. Thus, we conclude that ILP is not only well fit for the applications we consider, it is crucial that a technique of comparable power be used.

\section{Simple bridges between ILP and relational databases}

In this and the following sections, we discuss several ways in which ILP systems can be linked to databases, in increasing order of adaptation of the ILP system to the database. We first consider some rather trivial methods.

\subsection{Conversion of data}

ILP systems use logic to represent the data, the hypotheses, etc. The data are usually presented to the system in Prolog syntax, i.e. as a text file containing a number of facts in the form pred $\left(\arg _{1}, \ldots, \arg g_{n}\right)$.

When the data are available in a relational database, they can easily be transformed to Prolog syntax (for each relation, a set of facts is written with each fact corresponding to one tuple), and are then ready to be processed by an ILP system. This transformation can be seen as a pre-processing tool for the ILP system. This is the easiest way to use ILP for knowledge discovery in a relational database: nothing has to be changed to the ILP engine itself.

\subsection{A link to databases at the Prolog level}

As they work with a logical representation, many ILP systems are implemented in Prolog (e.g. Claudien[1], ICL[2]), or, when they are implemented in another

${ }^{1}$ It should be mentioned here that most ILP systems work with positive and negative examples, while in a database only positive examples are given. The closed world assumption has to be made in this case. Recently, however, more attention has been given to ILP systems that learn from positive examples only (this is often called learning in a non-monotonic setting; see for instance [1]). These systems are the ones for which the analogy holds best. 
language, use libraries that implement many features of a Prolog system (e.g. [9]). Some Prolog systems can be linked to relational databases. This means that part of the knowledge base is not read from a Prolog file, but is assumed to be in the database to which the Prolog system is linked. The internal database of the Prolog system is then augmented with the external database in a transparent way. When a literal $p(a, b)$ occurs somewhere, and the predicate $p$ corresponds to a relation $P$ in the relational database, then Prolog will send a query to the relational database system to determine wether the tuple $(a, b)$ is in relation $P$. If this is the case, the literal succeeds; otherwise it fails.

When an ILP system runs in a Prolog environment that supports this kind of link, it is not necessary to write the data in the database to a text file in the correct format, and then load this file into the system; it is sufficient to tell the system that those predicates can be found in the relational database.

This method to use ILP for knowledge discovery in a relational database is very straightforward; if the Prolog system underlying the ILP system supports links with database systems, the ILP system can work with the database without any changes at all, except some declarations to open and close the database. If the ILP system is not implemented in Prolog, or if the underlying Prolog system does not support links with databases, a significant extension of the system is of course required.

When compared with the first method, this method has the disadvantage that it is probably slower, as access to a relational database is needed for every literal that is to be tested, while with the first method one conversion is done beforehand, and afterwards all the data will be loaded in memory. On the other hand, a database may be too large to be loaded into the Prolog system; in that case, this method offers a simple alternative.

\subsection{A link to databases at the clause level}

We know that there is a straightforward relationship between first order logic and relational databases: a predicate is just a relation between its arguments. Similarly, there is a relationship between logical clauses and relational database queries (at least for the usual query languages, e.g. relational algebra, SQL). For ease of discussion, we will restrict ourselves here to the SQL query language.

In a clause, every literal corresponds to a tuple of a relation. The conjunction of a number of literals can be computed as a join, where the conditions on the join are given by the variables that the literals have in common. For instance: the query

?- $\operatorname{parent}(\mathrm{X}, \mathrm{Y}), \operatorname{parent}(\mathrm{Y}$, 'Laure').

is written in SQL as

SELECT P1.parent, P1.child

FROM PAREITT P1, P2

WHERE P1.child = P2. parent AND P2. child = 'Laure' 
This shows that logical clauses can easily be transformed into relational algebra formulas or SQL-statements. Discovery systems exploiting this property already exist; see e.g. [7, 8], where a coupling between the ILP-system RDT [6] and the Oracle database system is described. The resulting RDT/DB system makes use of SQL-queries akin to those given further in this paper. Recently, a preliminary version of a coupling between CLAUDIEN and Oracle has also been implemented.

ILP systems, when traversing the search space, check clauses for correctness, and associate heuristic values with them. In the characteristic setting, a clause $B \leftarrow H$ is correct if the query $\leftarrow B \wedge \neg H$ fails. When a clause is incorrect, a heuristic value can be given to it, indicating how close to correctness it is.

The heuristic value of a clause

$$
P(\ldots) \leftarrow Q_{1}(\ldots), \ldots, Q_{m}(\ldots)
$$

is usually computed as a function $f(p, n, c)$ where $p$ is the number of positive examples predicted by the clause to be positive, $n$ is the number of negatives predicted to be positive, and $c$ is the complexity of the clause. It is clear that a clause is closer to correctness when $n$ is smaller; also the usefulness of the clause increases with $p$ (a clause that predicts many positive examples is better than one that predicts few, even if both are correct, i.e. predict no negative examples to be positive).

The computation of $p$ and $n$ is normally done by the ILP system itself. The Claudien system, for instance, which is implemented in Prolog, will count how many times the body succeeds (by backtracking over the body until all solutions have been found), and how many times the body and head succeed together. If we call these numbers $s_{b}$ and $s_{b h}$, then this system computes $p$ and $n$ as $\hat{p}=s_{b h}$ and $\hat{n}=s_{b}-s_{b h}$. In fact, $p=\hat{p}$ and $n=\hat{n}$, only if all the literals in the clause are determinate; otherwise, the number of positive and negative substitutions of the clause are counted, and several substitutions can be found for one example. ${ }^{2}$ But for the purpose of using them in a heuristic, $\hat{p}$ and $\hat{n}$ work well enough.

Now, if we link the ILP system to a database, all the data are stored in relations, and one relation corresponds to each predicate. The numbers $s_{b}$ and $s_{b h}$ can then be obtained in SQL using the following queries:

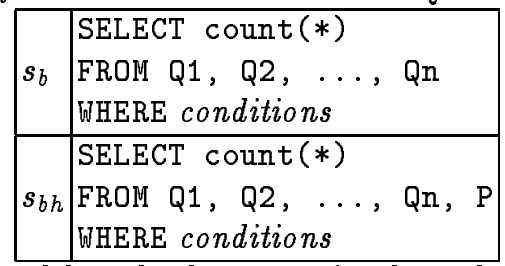

Although these queries have been written with the CLAUDIEN setting in mind, they can easily be adapted in such a way that they count examples, not substitutions, by changing the queries into:

${ }^{2}$ Counting examples is not appropriate in the CLAUDIEN context, where the head of a clause can consist of several literals; the meaning of the term "example" is therefore hard to define here. 


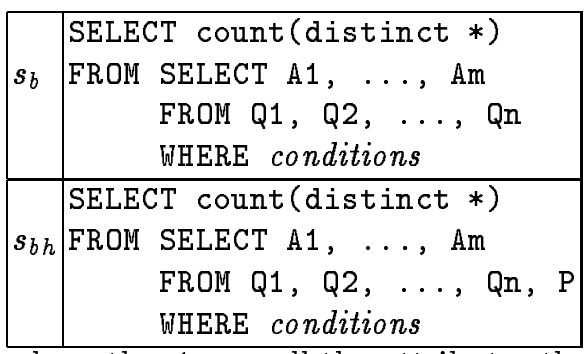

where the $A_{i}$ are all the attributes that occur in $P$.

In both cases, the second query will of course be computed using the intermediate relation computed by the first one; the work of joining the $Q_{i}$ should not be redone. Also note that SQL does not only compute the relations that contain the solutions to the queries $B$ and $B \wedge H$; it immediately counts the tuples in them. This way, the heuristic value of the clause is computed by the database system itself.

If we compare this approach with the previous one (a link to databases on the Prolog level), then there is a considerable efficiency gain:

- Instead of using the database server for each literal in the clause, and backtracking over the clause, which results in a very large number of database accesses, the database is only accessed once for each clause that is to be investigated. This results in much less overhead.

- Database systems have many ways of optimizing queries that generally are not used by Prolog compilers (e.g. performing the most selective queries first, using advanced indexation techniques, ...). This means that the heuristic value can in most cases be computed much more efficiently by the database system than could be done in Prolog.

When compared with the first approach (loading all the data into the Prolog system), there is of course a small disadvantage here, because a database has to be accessed (i.e. data are retrieved from secondary storage instead of main memory), but for large databases, we expect this disadvantage to be outweighed by the efficiency gain obtained by letting an advanced database system compute the heuristic values instead of implementing this in Prolog. We have not yet been able to confirm this experimentally, however.

\section{Redefining the search space}

In the previous sections it was shown that the link between ILP and a relational database can be established on the data level (conversion of data to logical representation), on the Prolog level (database access is completely transparant to the Prolog user), and on the level of the ILP system (transforming whole clauses into SQL queries).

We now go one step further, and in fact hereby leave the area of ILP. What we propose, is that the ILP techniques can be lifted to a search space consisting of database queries, instead of logical clauses. In other words, the discovery system 
does not use any logical representations anymore, and therefore strictly should not be called an ILP system; but it still uses essentially the same techniques.

In this section, we show how a hypothesis space consisting of relational algebra expressions can be generated and traversed. This section contains mainly ideas for future work; an algorithm is presented, but it has not been implemented yet.

\subsection{Some results from relational algebra}

We first mention some results known from database theory that will be useful later on. These results can be found in most standard works on database theory, e.g. [4].

Several operators are defined in the relational algebra, but it can be proven that they can all be defined using the following five operators (see also [10]):

- the cartesian product of two relations $(R \times S)$

- the union of two relations having the same arity $(R \cup S)$

- the projection of a relation on certain attributes $\left(\pi_{i_{1}, \ldots, i_{n}}(R)\right)$

- the selection from a relation of those tuples for which the value of two attributes $i_{1}$ and $i_{2}$ are the same $\left(\sigma_{i_{1}=i_{2}}(R)\right)$

- the selection from a relation of those tuples for which the value of two attributes $i_{1}$ and $i_{2}$ are different $\left(\sigma_{i_{1} \neq i_{2}}(R)\right)$

(We will assume that in a relation, attributes are referred to using the number of the column corresponding to the attribute; e.g. $\sigma_{1=3}$ selects from a relation those tuples which have the same values for the attributes in column 1 and column 3 . This way, we can avoid possible problems with renaming of attributes that are irrelevant to our discussion.)

The above implies that there is no loss of generality if we restrict ourselves to expressions that make use of only these operators. It is possible to go even further: every expression using these five operators (and therefore every expression using any relational algebra operator) can be written as a succession of cartesian products, selections, projections and unions, in this specific order. This follows from the following properties:

- if $\pi_{A}(R \cup S)$ is defined, then $\pi_{A}(R) \cup \pi_{A}(S)$ is defined and $\pi_{A}(R) \cup \pi_{A}(S)=$ $\pi_{A}(R \cup S)$

- if $\sigma_{C}(R \cup S)$ is defined, then $\sigma_{C}(R) \cup \sigma_{C}(S)$ is defined and $\sigma_{C}(R) \cup \sigma_{C}(S)=$ $\sigma_{C}(R \cup S)$

- if $(R \cup S) \times T$ is defined, then $(R \times T) \cup(S \times T)$ is defined and $(R \times T) \cup(S \times T)=$ $(R \cup S) \times T$

- if $\sigma_{C}\left(\pi_{A}(R)\right)$ is defined, then $\pi_{A}\left(\sigma_{C}(R)\right)$ is defined, and $\sigma_{C}\left(\pi_{A}(R)\right)=\pi_{A}\left(\sigma_{C}(R)\right)$

- if $R \times \pi_{A}(S)$ is defined, then there exists an $A^{\prime}$ such that $\pi_{A^{\prime}}(R \times S)$ is defined, and $R \times \pi_{A}(S)=\pi_{A^{\prime}}(R \times S)$.

- if $R \times \sigma_{C}(S)$ is defined, then $\sigma_{C}(R \times S)$ is defined, and $R \times \sigma_{C}(S)=\sigma_{C}(R \times S)$. 
The first three properties show that the union operator can always be moved outwards, the following two show that the projection operator can always be moved outwards but not past the union operator, and the last property shows that selection can be moved outwards but not past the projection or union operator. After all the operators have been moved, and considering the fact that consecutive projections can be written as one projection and consecutive selections as one selection, an expression of the form

$$
\bigcup_{i} \pi_{A_{i}}\left(\sigma_{C_{i}}\left(R_{i, 1} \times \cdots \times R_{i, n_{i}}\right)\right)
$$

emerges.

This property is important because it allows to reduce the size of the search space without giving up completeness. If the search space contains expressions where the operators can occur in any order, then many expressions are investigated that are actually equivalent, but written in a different way. This means that a lot of redundant computations are done. If only expressions of the above form are considered, this redundancy is removed, while the completeness of the search space is preserved.

Note that we write the expressions in this way, only in order to define the search space. When the expression is actually computed for some database instance, the order of the operators will be changed by the database system itself, in such a way that the result can be computed as efficiently as possible. In other words, the database takes care of the efficient computation of one single expression automatically; what we need to worry about, is that no redundant expressions are generated.

\subsection{An algorithm for finding relationships between relations}

ILP systems typically find a set of rules of the form

$$
p\left(X_{1}, \ldots, X_{n}\right) \leftarrow L_{1}, \ldots, L_{n}
$$

where each rule predicts some facts for $p$, and the set as a whole predicts all the facts of $p$. With this kind of rule corresponds one specific kind of relationship between a relation $R$ (predicate $p$ ) and a set of relations $R_{i}$, namely the fact that for some expression $E\left(R_{1}, \ldots, R_{n}\right)$, it holds that $E\left(R_{1}, \ldots, R_{n}\right) \subseteq R$. A program consisting of a set of rules $r_{i}$ is then equivalent to the union of the $E_{i}$ corresponding to each $r_{i}$. If the program is complete, then $\bigcup_{i} E_{i}\left(R_{i, 1}, \ldots, R_{i, n_{i}}\right)=R$.

The algorithm in Figure 1 searches for all the expressions $E_{i}$ such that

$$
E_{i}\left(R_{1}, \ldots, R_{n}\right) \subseteq R
$$

given as input $R$ and $\mathcal{R}=\left\{R_{1}, \ldots, R_{n}\right\}$; i.e. it finds a number of (possibly overly specific) descriptions of $R$. These expressions are all of the form $\pi_{A}\left(\sigma_{C}\left(R_{i, 1} \times\right.\right.$ $\left.\cdots \times R_{i, n_{i}}\right)$ ).

The algorithm takes some parameter $N$ as an upper bound on the complexity of the expressions that can be derived. The parameters that define the kind of 
expressions that can be derived, all together, are called the language bias of the algorithm. For this algorithm, $N$ is part of the language bias, as well as the set $\mathcal{R}$.

An exact definition of $R$, if one exists, can be among the $E_{i}$. If not, $R$ might still be expressible as the union of some or all of the $E_{i}$. If the union of all the $E_{i}$ still produces a proper subset of $R$, then $R$ cannot be defined within the language bias used. Since the search space is complete (with respect to given complexity bounds), it follows that if there exists a relationship between $R$ and $\mathcal{R}$ that is expressable in relational algebra, it will be found.

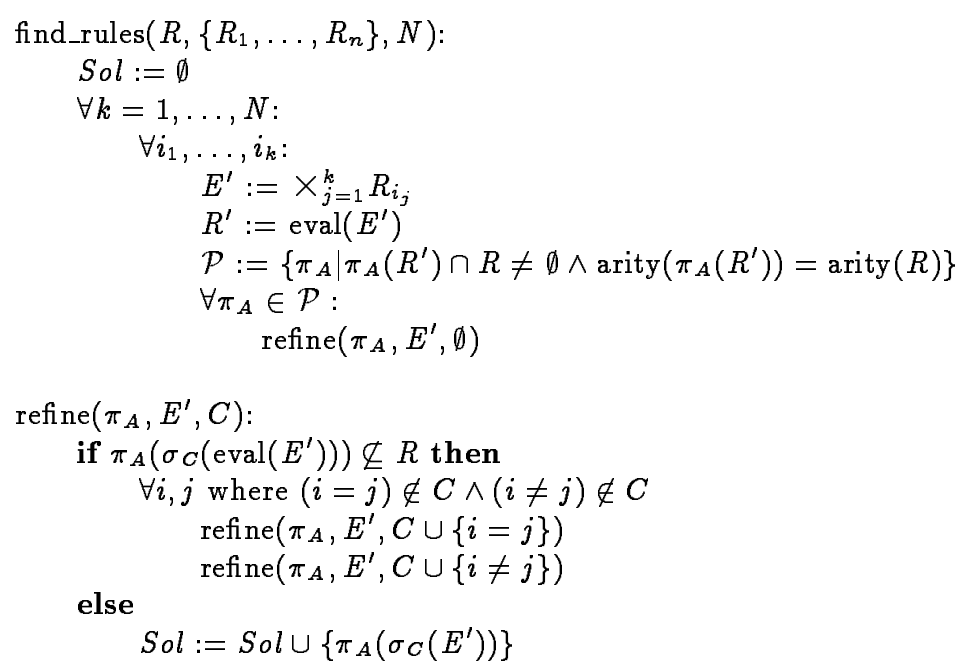

Fig. 1. An algorithm for finding relationships between relations

Algorithm 1 works as follows. It considers all subsets of $\mathcal{R}$ of cardinality at most $N$. For each such set, it computes the cartesian product of all the $R_{i}$ in the subset, and finds all the projections $\pi_{A}$ onto $r$ attributes (with $r$ the arity of $R$ ) for which the resulting relation has at least one tuple in common with $R$. (If it has no tuples in common with $R$, then it is not possible to find a subset of this relation that is also a subset of $R$, except the empty relation, which is a trivial case.)

Note that the algorithm will compute expressions, not the resulting relations; therefore, when we write $E^{\prime}:=\langle$ expression $\rangle$, this means that the expression itself is stored in $E^{\prime}$. The evaluation of an expression, when needed, is denoted explicitly using the eval function.

For each $R^{\prime \prime}$ for which $R^{\prime \prime} \cap R \neq \emptyset$, it may be possible, using the selection operator $\sigma$, to find a non-empty subset of $R^{\prime \prime}$ that is also a subset of $R$. However, this selection operator should be applied before the projection operator, not 
afterwards, in order to preserve the completeness of the search space.

The refine procedure in Figure 1 finds every selection $\sigma_{C}$ for which $\pi_{A}\left(\sigma_{C}\left(\times_{i} R_{i}\right)\right) \subseteq$ $R$, and $C$ is a minimal set of conditions (i.e. leaving out one condition from $C$ will not produce a subset of $R$ ). For each $\sigma_{C}$ for which this holds, the expression $\pi_{A}\left(\sigma_{C}\left(\times_{i} R_{i}\right)\right)$ is added to the solution set $S o l$.

This algorithm is written from the point of view of characteristic learning: it returns every expression with which a non-empty subset of $R$ is computed. When the aim is simply to define $R$ in terms of the other relations, using as simple an expression as possible, one can of course first generate all these expressions and then find a minimal subset of them such that the union of the expressions in the subset is complete; but this method, although correct, is quite inefficient. A covering approach, as followed by many ILP algorithms, is often preferable. The algorithm can easily be adapted to this end: using a FOIL-like covering approach, it would select at each time that expression that results in as large a subset of $R$ as possible (not counting the tuples that were already produced by other expressions), and repeatedly traverse the search space in search for such an expression until $R$ has been covered completely. The use of heuristics to guide this search can also easily be incorporated.

\subsection{Some comments}

Although the above algorithm was given for relational algebra, it is obvious that a similar algorithm can be given for SQL statements. An algorithm searching an SQL search space would be of more practical value than one using relational algebra, as most database systems use SQL, not relational algebra. Therefore, an SQL using equivalent of Algorithm 1 seems more worthwhile to be implemented than the algorithm itself. SQL, however, offers many more possibilities than relational algebra, e.g. grouping constructs, counting, ... It seems that a good way to investigate SQL search spaces would be to start from constructs that correspond to those offered by the relational algebra, and then adding more features.

When we take a look at the approach proposed here, some interesting topics are raised:

- The proposed algorithm uses a very simple language bias: there is simply a bound on the complexity, and a specification of the relations that can be used. More elaborate language specifications could be based on those used in ILP systems (e.g. $\mathcal{D}$ LAB [3]), but there will probably be enough differences to justify some specific research on, for instance, language specifications for SQL.

- The search space is expected to be organized differently than the clausal logic search spaces. For instance, adding a literal to a clause is considered to be a refinement step in ILP, but in the algorithm we propose, the relations to be used are chosen in advance, and refinement from then on consists of adding selection operators (in logic, this corresponds to unifying variables or adding the condition that they should not be equal). The queries are 
equivalent with logical clauses, but ordered differently. This may result in other solutions being found, if only a subset of all the rules is to be returned (as with the covering approach).

- The notion of simplicity of an expression is different in relational algebra than in logic. For instance, when the same variable occurs several times in a logical clause, this is not considered to make the clause more complex (one could even argue that the clause is less complex, as it uses less variables). In relational algebra, on the other hand, unification of variables corresponds to an explicit condition that two values must be equal, and the addition of this condition makes the clause more complex. This is one example of the fact that desirable properties of algebraic expressions may differ from those of logical clauses, and that it is better to work with the expressions themselves instead of with clauses that are equivalent to them.

- Relational algebra is less expressive than first order logic, because there is no equivalent for recursive definitions. We consider this not to be a problem, however, because non-recursive query language such as SQL are used very frequently to query relational databases, and for most databases they have enough expressive power. Moreover, a recursive logical clause cannot be transformed to SQL, so even if a relation could be defined recursively in terms of other relations and itself, there would be no way to write an SQL-view defining this relation.

\section{Conclusions}

Finding an intensional definition for a relation, or investigating which integrity constraints hold in a database, are knowledge discovery tasks that bear a large resemblance to typical ILP classification or discovery tasks. Therefore one expects that ILP-techniques are very suitable for this kind of knowledge discovery.

We have given an overview of several ways in which ILP systems can be linked to relational databases, in order to solve the problems mentioned. These methods range from trivial conversion of relational data to logic, to transferring existing ILP techniques to other domains. We have also discussed some advantages and disadvantages of the different approaches. We are convinced that the more sophisticated approaches will offer several advantages, one of which is efficiency; and this supports our belief that ILP techniques are useful for knowledge discovery in large databases.

Although this paper has only considered some rather specific knowledge discovery tasks, we believe that the advantages discussed here will hold in general, and that efficient methods for general-purpose knowledge discovery in databases can easily be derived from the existing ILP-techniques, without any need for the development of algorithms specifically designed for one task. 


\section{Acknowledgements}

Hendrik Blockeel is supported by the Flemish Institute for the Promotion of Scientific and Technological Research in the Industry (IWT). Luc De Raedt is supported by the Belgian National Fund for Scientific Research. This work is also part of the European Community Esprit project no. 20237, Inductive Logic Programming 2.

\section{References}

1. L. De Raedt and L. Dehaspe. Clausal discovery. Forthcoming, 1995.

2. L. De Raedt and W. Van Laer. Inductive constraint logic. In Proceedings of the 5th Workshop on Algorithmic Learning Theory, Lecture Notes in Artificial Intelligence. Springer-Verlag, 1995.

3. L. Dehaspe and L. De Raedt. DLAB: a declarative language bias for concept learning and knowledge discovery engines. Technical Report CW-214, Department of Computer Science, Katholieke Universiteit Leuven, October 1995.

4. R. Elmasri and S. B. Navathe. Fundamentals of Database Systems. The Benjamin/Cummings Publishing Company, 2nd edition, 1989.

5. U. M. Fayyad and R. Uthurusamy, editors. Proceedings of the First International Conference on Knowledge Discovery and Data Mining. AAAI Press, August 1995.

6. J-U. Kietz and S. Wrobel. Controlling the complexity of learning in logic through syntactic and task-oriented models. In S. Muggleton, editor, Inductive logic programming, pages 335-359. Academic Press, 1992.

7. G. Lindner. Anwendung des lernverfahrens RDT auf eine relationele datenbank. Master's thesis, Universität Dortmund, August 1994. In German.

8. G. Lindner and K. Morik. Coupling a relational learning algorithm with a database system. In Y. Kodratoff, G. Nakhaeizadeh, and C. Taylor, editors, Workshop Notes of the MLnet Familiarization Workshop on Statistics, Machine Learning and Knowledge Discovery in Databases, pages 163-168, 1995.

9. S. Muggleton. Inverse entailment and progol. New Generation Computing, 13, 1995.

10. J. Paredaens. On the expressive power of the relational algebra. Information Processing Letters, 7(2):107-111, February 1978.

11. J.R. Quinlan. FOIL: A midterm report. In P. Brazdil, editor, Proceedings of the 6th European Conference on Machine Learning, Lecture Notes in Artificial Intelligence. Springer-Verlag, 1993.

This article was processed using the $\mathrm{I}_{\mathrm{E}} \mathrm{TX}$ macro package with LLNCS style 